\title{
Where has all the information gone?
}

\author{
H. D. Zeh \\ Universität Heidelberg \\ www.zeh-hd.de
}

\begin{abstract}
The existence of spacetime singularities is irrelevant for the irreversible appearance of black holes. However, confirmation of the latter's unitary dynamics would require the preparation of a coherent superposition of a tremendous number of appropriate "Everett worlds".
\end{abstract}

Key words: black holes, quantum indeterminism, decoherence PACS:

\section{The information loss "paradox"}

Stephen Hawking's claim of a lost bet [1] recently stirred up a lot of interest and discussion in the media (physical journals included). If correct, it would mean that the information absorbed by a black hole must later be emitted in some way, for example by means of correlations existing within the Hawking radiation - even though they can hardly ever be used to recover the original information. The opposite assumption that this information is irretrievably lost, while the black hole may completely disappear, is generally regarded as a paradox, since it would violate unitarity $[2,3,4,5]$.

Hawking's new arguments against his own bet rely on a detailed (though not yet published) calculation, which must use certain assumptions and approximations - they are not based on any novel empirical evidence. Therefore, his result must simply reflect these assumptions, regardless of whether or not they are explicitly stated. For example, if unitarity is presumed for the underlying theory, Hawking's claim does not need any further calculation [6]. Similarly, disappearance of "information" would be unavoidable if a classical spacetime that contained future singularities were assumed to correctly describe the real world. 
However, exact classical spacetimes (or gravitational fields) are known to be inconsistent with the presence of quantized matter $[7,8]$. According to canonical quantum gravity they are even excluded in the same way as particle trajectories are in quantum mechanics. Opposite conclusions about the (ir)reversibility of black hole dynamics thus arise from different beliefs regarding the universal validity of quantum theory.

Albert Einstein's general relativity requires the existence of spacetime horizons. They lead to the remarkable consequence that quantum entanglement, which again Einstein brought into particular focus in his paper with Podolsky and Rosen [9] (albeit in order to prove quantum theory incomplete), must arise between the inner and outer regions of a black hole. This leaves the corresponding "information" drastically nonlocal, that is, neither inside nor outside. John Bell's analysis and subsequent experiments have demonstrated that entanglement must be part of reality rather than being the consequence of an incomplete description (mere statistical correlations).

The dispute about the nature of an information loss (or entropy increase) is not at all new. The conflict between deterministic laws and irreversible phenomena occurred on many occasions. Let me therefore emphasize that information is here usually defined by means of a formal ensemble of possible states which are assumed to evolve in time according to some dynamical law. For example, information would be conserved under deterministic (or unitary in the case of quantum theory) laws. The problem then regards such (always hypothetical, though in most cases empirically verified) laws - not what we happen to know, or are able to use, observe or calculate.

\section{Information loss in classical statistical physics}

Many deviations from information-conserving laws are meaningful and successfully used. For example in classical physics, all master equations, such as Boltzmann's collision equation, are based on the permanent neglect of arising correlations (or of other kinds of "irrelevant information") when calculating into the future direction of time. The applicability of this very restrictive assumption, which requires a special initial condition for our world, is responsible for the observed increase of phenomenological entropy. By definition, ensemble entropy is conserved under deterministic equations of motion if it is calculated - in contrast to phenomenological entropy - by taking into account all irrelevant information, such as correlations. This would require a highly non-extensive concept of entropy,

$$
S=-k_{B} \int \rho(p, q) \ln \rho(p, q) d p d q
$$


which is a functional of the density $\rho(p, q)$ in many-particle (or any other canonical) phase space. Statistical correlations between local objects at different positions are non-local, that is, they are themselves at no place. Only if $\rho$ approximately factorizes into a mean spatial density and the rest does one obtain a spatial entropy density, which allows entropy to flow in space (as phenomenologically required) [10]. The observed entropy increase reflects the transformation of part of the conserved information into information about irrelevant properties, such as uncontrollable correlations. The latter are usually neglected by the concept of physical (local) entropy. However, the real (completely defined) physical state does not contain any statistical correlations.

Correlations propagate and multiply very efficiently by interactions with the environment - either by means of chains of Boltzmann type collisions between molecules, or by (even very weak) long range interactions, since the latters' effect on the molecules of a distant gas is usually strongly amplified by subsequent molecular collisions [11]. Hence, in a "causal" world, where all correlations require local causes in their past, the information they may represent is irreversibly lost for all practical purposes. The deterministic evolution of most subsystems of the universe depends crucially on the (otherwise irrelevant and therefore unknown) precise physical states of their global environments - even though determinism is classically well defined in principle by the presumed global dynamics. An exact reversal of motion (or a "complete recovery of information") for any macroscopic system would therefore require a precise reversal or recurrence of the state of the whole universe.

\section{3 "Information" in quantum theory}

In quantum theory, global unitarity would similarly warrant the conservation of global entropy or lacking "information" (negentropy) if this is now defined by the functional

$$
S=-\left(I-I_{0}\right)=-k_{B} \operatorname{Trace}(\rho \ln \rho)
$$

of a global density matrix $\rho$. However, quantum theory is usually understood as an indeterministic (probabilistic) theory. It is this contrast which forms the most fundamental information loss paradox in physics.

If one now assumed (with Bohr) that quantum concepts were not applicable to macroscopic objects, unitarity would not even be an issue for them. If one assumes instead (with von Neumann, Pearle or Ghirardi) that quantum concepts are universal, while the Schrödinger equation has to be modified in order

to describe the collapse of the wave function, unitarity is an approximation 
- valid only for microscopic objects. However, if the Schrödinger equation is assumed to be exact, one has to conclude that there is a superposition of myriads of branching Everett "worlds". The time arrow of this branching must again be the consequence of a cosmic initial condition (for the universal wave function). Our observed universe would then be represented by one single, dynamically autonomous branch of the global wave function that permanently splits further by means of the dislocalization of quantum phases (decoherence [12]). Considered by itself, this branch describes the same stochastic phenomena as a genuine collapse (that is, quantum measurements in a general sense). According to this description, part of the information about the initial global state is deterministically transformed into inaccessible quantum correlations between different Everett branches, while the growing specification of the observer's branch (characterized by all previous "measurement results") describes in principle a (usually negligible) entropy decrease. So the crucial question is: What precisely is the global density matrix used in the above definition of entropy? In practice, it is always based on a coarse graining of locality as well as on an effective collapse (Everett branching).

Quantum correlations (entanglement) are very different from classical statistical correlations. This becomes most obvious when one attempts to trace back in time the quantum state of a system by using the new information gained in a measurement ("postselection"). While in classical physics this procedure would improve knowledge (reduce an ensemble) also about the past, in quantum theory the postselected state, if calculated backwards in time, is in general incompatible with the documented history. The reason is that a quantum density matrix does not simply represent an ensemble (a probability distribution) of possible states - that is, not just incomplete information. Quantum correlations characterize superpositions, which depend on the phases of their complex coefficients. They define individual physical states. For example, two particles with definite total spin are in a superposition of product states by means of Clebsch-Gordon coefficients (even when at very different locations). A collapse of this wave function into a product of states would violate angular momentum conservation. The term "quantum information", though intuitively appealing because of certain analogies with the classical case, should therefore be used with great care in order to avoid misconceptions. Nonetheless, entanglement is the essential concept underlying the information loss paradox of black holes.

This "loss of quantum information" occurs in particular in the form of decoherence. Thereby, quantum correlations with an uncontrollable environment are produced in an irreversible manner (in formal analogy to Boltzmann correlations in classical mechanics). Their phase relations are then irrelevant for all local observers, who, for the same reason, would not possess any physical state by their own any more. This gives rise to an effective ensemble of local "states of being conscious" for each of them, existing in different Everett "worlds". The various states of different observers are thereby correlated by means of 
their specific entanglement.

In this way, outcomes of measurements or other consequences of decoherence are objectivized, and may as well be described by stochastic dynamics (effective master or quantum Langevin equations). Without any explicit dynamical description of measurements, there would be "no measurement problem, as there is no theory" [13]. The identification of a specific result by the subjective observer may then be regarded as its selection from the apparent ensemble that was created by decoherence. It does not represent the selection of a subensemble from a pre-existing ensemble (a mere "increase of information"). For this reason, the ensemble entropy of the global system may be smaller than the formal entropy of any of its local subsystems even in the case of complete global information (that is, a pure global state) - see Chap. 2 of [12].

In scattering experiments, the unitary quantum description is usually interrupted at the detector. This comes very close to the Copenhagen interpretation, although it can be justified by the unavoidable (unitary) interaction of the macroscopic detector with its environment. Even the macroscopic parts of this undoubtedly real object rely in an essential way on quantum theory. The pragmatic attitude may work for practical purposes - but it is clearly not conceptually consistent. It is this conceptual defect that is usually attributed to an "absence of microscopic reality" (or of a "quantum world"). In particular, detectors and other macroscopic objects, though strongly entangled with their environments, may fall into black holes!

\section{Information Loss in General Relativity}

In general relativity, matter may fall onto singularities within finite proper times, whereby all information about its state would become meaningless (or disappear). On the other hand, simultaneities, on which the global states are defined, may now be chosen in such a way that they never reach the local singularity for coordinate values corresponding to all finite times in the asymptotic region (see Fig. 1). This choice should not affect the physics and its correct description in regions of space where differently chosen simultaneities coincide.

The latter expectation would not represent a conceptual problem if physics were local. The real (completely defined) external state of a black hole, say, would then exist independently of the internal one, while correlations could affect only a statistical (incomplete) description.

In nonlocal quantum theory, however, not only the state of matter on the singularity, but also its entanglement with that in the regular region is lost. Although it may be tempting to regard the singularity as an "ultimate envi- 


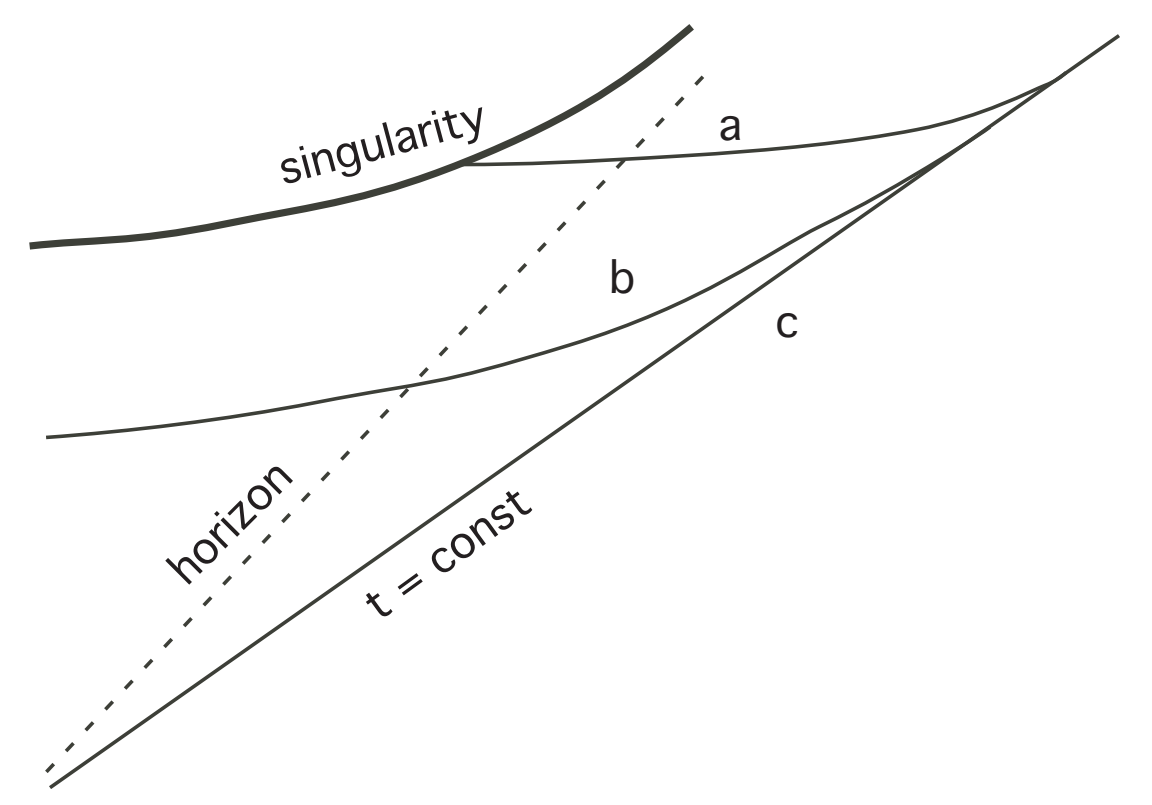

Fig. 1. Various simultaneities for a black hole in a Kruskal type diagram: (a) hitting the singularity, (b) entering the regular interior region only, (c) completely remaining outside (Schwarzschild time coordinate $t$ ). Schwarzschild time is appropriate in particular for posing asymptotic boundary conditions. The angle between the horizon and the line $t=$ const can here be arbitrarily changed by a passive time translation. This includes the (apparently close) vicinity of the horizon, which can thus be arbitrarily "blown up" in the diagram - thus focussing consideration on the distant future.

ronment" (which might explain a genuine collapse of the wave function), the free choice of simultaneities demonstrates that this is by no means conclusive. Even the global vacuum contains entanglement between both sides of a horizon, locally giving rise to thermal Hawking or Unruh radiation (that is, with or without a singularity). Since the reduced density matrix that describes local systems at some distance from the horizon cannot depend on the continuation of the simultaneity inside the horizon or in its close external vicinity, it suffices that the "information" corresponding to these quantum correlations is lost for all practical purposes of distant observers. In principle, there is then no difference to conventional decoherence (without black holes) [14]. Because of the extreme time dilation, even the close external vicinity of the horizon can causally affect distant systems, such as external observers, only in the very distant future.

Since the value of Schwarzschild time $t$ (the arcus tangens of the angle at which it appears in the figure) has no absolute meaning, the external part of the diagram remains valid far into the future - including times when the black hole should have disappeared by means of Hawking radiation from the point of view of an external observer. He would observe the black hole disappear even before (according to these Schwarzschild simultaneities) a horizon has formed. The mixed state describing the radiation (a global ensemble) is then created 
only by the usual statistical treatment of the radiation process (cf. [6]).

Infalling matter would in turn be affected by cosmic events or boundary conditions in the distant future if the thermodynamical arrow of time ("causality") ever reversed its direction during the cosmic evolution. Because of the diverging time dilation this would allow the matter just in time to feel advanced radiation and experience recoherence in order to retro-cause the black hole to grow hair and expand again. For example, a time-symmetric version of Penrose's Weyl tensor condition [15] (a vanishing Weyl tensor on all singularities) would entirely eliminate all inhomogeneous singularities (such as those of black holes) together with their event horizons, without essentially affecting the state of our present universe far from black holes [16,17]. Global "information" (taking into account all correlations) could then be conserved under a Schrödinger equation and for all choices of simultaneities.

It seems that our conventional interpretation of black holes owes too much to the classical picture that "nothing unusual happens" at the horizon. While this would remain true under a purely local consideration, observers orbiting and approaching a galactic black hole, for example, might have sufficient time to observe (and get affected by) much of the future cosmic history as in an extreme quick motion movie - provided they can survive the novel affects caused on them by the external world. However, double-ended boundary conditions, such as a symmetric Weyl tensor condition, may not be dynamically consistent [18] with classical general relativity, which allows free initial or final conditions only.

\section{Quantum Gravity}

The information loss paradox is usually discussed in connection with quantum gravity. Otherwise the question of unitarity would not make sense for black holes as geometrodynamical objects. However, regarding black holes as isolated quantum systems obeying a Schrödinger equation would repeat the popular mistake of describing a quantum measurement according to von Neumann as an isolated unitary interaction between the measured system and a macroscopic device. In both cases, decoherence by the environment is a crucial part of the story.

Just as von Neumann's measurement interaction does not depend on any details of the apparatus, the key argument regarding black hole unitarity does not depend on the precise variables which describe quantum gravity - provided only the basic principles of quantum theory are maintained. The very idea of "quantization" can generally be understood as the conceptual reversal of decoherence: the re-introduction of those superpositions which are gener- 
ally suppressed by interaction with the environment, and which were therefore missing in the classical description. In general relativity, quantization thus leads to a wave functional on superspace (the configuration space of all spatial geometries, which form the kinematics of general relativity). It is quite irrelevant for the present discussion whether this configuration space may later have to be modified (or reconstructed) under more general mathematical considerations - for example in terms of a configuration space consisting of loop integrals or strings and other fields on some higher dimensional space instead of the traditional superspace including matter fields. All one needs is to take the wave function seriously [19]. There is neither a "crisis" nor an indication of a "paradigm shift" [20] that would not yet have occurred in a universally valid quantum mechanics. As usual, the paradox is an artifact of the insistence on classical concepts.

In particular, superpositions of different spatial geometries are permanently decohered by (become entangled with) matter. In this way, quasi-classical spacetimes emerge in the form of propagating, dynamically autonomous wave packets from the universal wave function [21]. Black holes even owe their timeasymmetric properties, including future horizons, to this embedding into their time-directed environment [14,22]. Since general relativity is time reversalsymmetric, energy eigenstates would have to be symmetric or antisymmetric superpositions of black and "white" holes [23], but would immediately decohere into their components in an irreversible manner if they ever came into existence. This establishes a superselection rule separating black and white holes, which, because of the long range of gravitational interactions, must even be correlated with the time arrow of the universe (analogous to correlations relating different observers of the same measurement). Therefore, only black holes can be observed. Interference experiments with black holes, just as with other macroscopic objects, would require the coherent preparation of many Everett branches (leading to their recoherence), while microscopic "virtual holes" would neither be black nor white. However, the structure of this Everett branching may appear very different to asymptotic observers and those being close to black hole horizons [24].

The deepest consequence of quantum gravity (and other reparametrization invariant theories) is the absence of any dynamical time parameter on a fundamental level. This requires that global quantum states must obey the Wheeler-DeWitt equation $H \Psi=0$. A concept of time describing a succession of states that, in particular, allows the formal distinction between initial and final conditions can then only be derived within the range of validity of a Born-Oppenheimer approximation with respect to the Planck mass [25,26]. Fundamental cosmic boundary conditions have instead to be postulated (or derived from new principles) for the timeless state $\Psi[27]$.

Unless otherwise enforced by means of such boundary conditions, this station- 
ary solution $\Psi$ of a real Wheeler-DeWitt equation must be real, too, and may in some regions of configuration space contain a factor $\sin (k a)$, characterizing the cosmic expansion parameter $a$. This is then usually decohered by its environment into components $e^{i k a}$ and $e^{-i k a}$, where the sign of $i$ has no physical meaning in the absence of any time dependence of the form $\exp (i \omega t)$. Therefore, there is no distinction between big bang and big crunch any more in quantum gravity. The Born-Oppenheimer approximation would break down close to a conceivable turning point of the cosmic expansion (where $\Psi$ describes geometries on Schwarzschild type simultaneities which contain spatial regions very close to black hole horizons) - thus undermining the concept of a universe evolving in time beyond its maximum extension while containing regions of high density which would classically very soon develop horizons and singularities.

Similar consequences would arise from all time-less low-entropy conditions at singularities, such as those excluding a singular Weyl-tensor. Other conditions to eliminate black hole singularities have recently been suggested within the framework of loop quantum gravity [28]. However, a quasi-classical spacetime (that is, a quasi-trajectory of spatial geometries) would have to be represented by an individual Everett branch of the wave function (or by a superposition

of branches which differ only by their quasi-classical matter variables). Therefore, these quasi-classical histories of geometry cannot separately obey unitary dynamics. In quantum gravity we are not allowed any more to ask: What happens at the horizon? but only: What is the structure of the wave function in the corresponding region of configuration space?

\section{References}

[1] S.W. Hawking, unpublished contribution at GR17 (Dublin 2004) - see "additions" to www.dcu.ie/ nolanb/gr17.htm.

[2] S.W. Hawking, Phys. Rev. 14 (1976) 2460.

[3] G. 't Hooft, Nucl. Phys. B 335 (1990) 138.

[4] D. Gottesmann, J. Preskill, JHEP 0403 (2004) 026.

[5] G.T. Horowitz, J. Maldacena, hep-th/0310281.

[6] D.N. Page, Phys. Rev. Lett. 44 (1980) 301.

[7] A. Einstein, in: Albert Einstein, Philosopher-Scientist, P.A. Schilpp (Ed.), The Library of Living Philosophers, 1949.

[8] D.N. Page, C.D. Geilker, in: Quantum Structure of Space and Time, M.J. Duff, C.J. Isham (Eds.), Cambridge UP, 1982. 
[9] A. Einstein, B. Podolsky, N. Rosen, Phys. Rev. 47 (1935) 777.

[10] H.D. Zeh, The Physical Basis of the Direction of Time, fourth ed., Springer, Berlin 2003 - see also www.time-direction.de.

[11] E. Borel, Le Hasard, Alcan, Paris, 1914.

[12] E. Joos, C. Kiefer, H.D. Zeh, D. Giulini, J. Kupsch, I.-O. Stamatescu, Decoherence and the Appearance of a Classical World in Quantum Theory, second ed., Springer, Berlin, 2003; M. Schloßhauer, Rev. Mod. Phys. 76 (2004) 1267.

[13] H.D. Zeh, Found. Phys. 1 (1970) 69.

[14] C. Kiefer, Class. Quant. Grav. 18 (2001) L151; and in: Decoherence and Entropy in Complex Systems, H.-T. Elze (Ed.), Springer, Berlin, 2004.

[15] R. Penrose, in: General Relativity, S.W. Hawking, W. Israel (Eds.), Cambridge UP, 1979.

[16] H.D. Zeh, in: Physical Origins of Time Asymmetry, J.J. Halliwell, J. PerezMercader, W.H. Zurek (Eds.), Cambridge UP, 1994.

[17] C. Kiefer, H.D. Zeh, Phys. Rev. D 51 (1995) 4145.

[18] H.D. Zeh, physics/0306083.

[19] H.D. Zeh, in: Science and Ultimate Reality, J.D. Barrow, P.C.W. Davies, C.L. Harper (Eds.), Cambridge UP, 2004; quant-ph/0204088.

[20] L. Susskind, hep-th/0204027

[21] E. Joos, Phys. Lett. A 116 (1986) 6.

[22] J.-G. Demers, C. Kiefer, Phys. Rev. D 53 (1996) 7050.

[23] P. Hájíček, C. Kiefer, Int. J. Mod. Phys. D 10 (2001) 775.

[24] H.D. Zeh, in: Old and New Questions in Physics, Cosmology, Philosophy, and Theoretical Biology, A. van der Merwe (Ed.), Plenum, 1983.

[25] H.D. Zeh, Phys. Lett. 116 (1986) 9.

[26] C. Kiefer, Quantum Gravity, Clarendon Press, Oxford, 2004.

[27] H.D. Conradi, H.D. Zeh, Phys. Lett. A 154 (1991) 321.

[28] A. Ashtekhar, M. Bojowald, gr-qc/0504029 